\title{
Minimal Solvers for Relative Pose with a Single Unknown Radial Distortion
}

\author{
Yubin Kuang $^{1,3} \quad$ Jan Erik Solem ${ }^{1,3} \quad$ Fredrik Kahl $^{1,2} \quad$ Kalle Åström $^{1}$ \\ ${ }^{1}$ Centre for Mathematical Sciences, Lund University, Sweden \\ ${ }^{2}$ Department of Signals and Systems, Chalmers University of Technology, Sweden \\ ${ }^{3}$ Mapillary AB, Sweden \\ \{yubin, solem, fredrik, kalle\}@maths.lth.se
}

\begin{abstract}
In this paper, we study the problems of estimating relative pose between two cameras in the presence of radial distortion. Specifically, we consider minimal problems where one of the cameras has no or known radial distortion. There are three useful cases for this setup with a single unknown distortion: (i) fundamental matrix estimation where the two cameras are uncalibrated, (ii) essential matrix estimation for a partially calibrated camera pair, (iii) essential matrix estimation for one calibrated camera and one camera with unknown focal length. We study the parameterization of these three problems and derive fast polynomial solvers based on Gröbner basis methods. We demonstrate the numerical stability of the solvers on synthetic data. The minimal solvers have also been applied to real imagery with convincing results ${ }^{1}$.
\end{abstract}

\section{Introduction}

Taking radial distortion into account is important in pose estimation and structure from motion problems. Problems for relative poses with unknown radial distortion have been studied extensively in the past [9, 1, 10, 15, 16, 14]. For all these cases, either constant or varying radial distortion is assumed on the cameras. Such settings are useful for selfcalibrating cameras with no prior knowledge on the camera intrinsics. Estimating relative pose and the radial distortion simultaneously is achieved by solving either a linear system or a polynomial system. Due to the difficulty of these problems, they either require many point correspondences or dedicated polynomial solvers. In fact, some minimal prob-

\footnotetext{
${ }^{1}$ This work was supported by the Swedish Research Council (grant no. 2012-4215 and grant no. 621-2009-4625) and the Swedish Foundation for Strategic Research, within the programmes RIT08-0043, ELLIIT and Future Research Leaders.
}
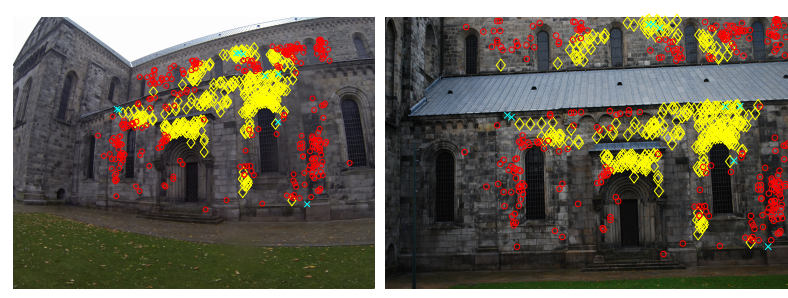

Figure 1. Example of modeling one-sided radial distortion for estimating relative pose. Left: an image taken with an GoPro-Hero3 with unknown radial distortion. Right: an image taken with a Nikon D60 camera which has known radial distortion. Yellow diamonds are the common inliers for fundamental matrix estimation obtained by both the standard 7-point method [12] and our minimal one-sided 8-point method. By considering radial distortion, our method obtains many extra inliers (red circles) while misses only a few inliers (cyan crosses) compared to the 7-point method.

lems of importance have still not been solved due to their difficulty, like estimating the relative pose with unknown focal length and radial distortion (both constant). In a general structure from motion pipeline, however, it is common that certain seed images have pre-calibrated or already known radial distortion. For these cases, the prior on the calibration of one of the cameras can reduce the complexity of the problem. In [5], a similar strategy has been applied in focal length estimation where the focal length of one camera is assumed to be known. This motivates us to study the problem of relative pose estimation with a single unknown radial distortion (sometimes referred to as one-sided problem). We are particularly interested in solving their related minimal problems for robust estimation purposes.

Related Work. To model radial distortion, we have followed the one-parameter division model proposed by Fitzgibbon [9] and assumed that the radial distortion center is known. For fundamental matrix and constant radial 
distortion on both cameras, Fitzgibbon proposed an overdetermined 9-point solver which involves solving a quadratic eigenvalue problem that has two solutions. The minimal case of this problem was solved in [15], and has in general 12 solutions. For a fundamental matrix with varying radial distortion, a linear solution using 13 point correspondences was proposed in [1]. An efficient and stable 9-point minimal solver was later derived in [14] with 24 solutions. For an essential matrix with constant radial distortion, which requires minimally 6 point correspondences, there are in general 56 solutions. It was also solved in [14]. The most related work to this paper is [4], which considered the onesided fundamental matrix estimation problem. In that paper, instead of solving the minimal case with 8 point correspondences, 9 point correspondences are used to simplify the problem into that of solving a cubic equation. While obtaining a simple overdetermined solver is useful, the study of the minimal cases is of both of theoretical interest and of great practical importance in robust estimation, for example using RANSAC [8]. Non-iterative solvers for estimating the radial distortion center (both one-sided and two-sided cases) were also studied in $[17,3,2]$. Even though the additional modeling of radial distortion center is useful, it has been shown that the estimation can be very sensitive to noise [3].

Contributions. In this paper, we study the three unsolved minimal cases for relative pose estimation with a single unknown radial distortion: (i) 8-point fundamental matrix and radial distortion, (ii) 7-point essential matrix, focal length and radial distortion, and (iii) 6 point essential matrix and radial distortion. For each of these cases, we derive a parameterization and a linear elimination scheme to simplify the polynomial systems. We study the polynomial systems and verify the number of solutions. We then develop fast and stable polynomial solvers for all these minimal cases. These solvers are minimal, fast and more accurate than previous non-minimal solvers, for example [4]. The availability of these fast solvers enables the possibility of initializing a large-scale structure from motion pipeline with radial distortion taken into account. We demonstrate the benefits of modeling radial distortion by using our proposed solvers.

\section{Problem Formulation}

We use the pinhole camera model and assume a oneparameter division model for radial distortion as in [9]. Under radial distortion, the relation between undistorted point coordinates $\mathbf{p}_{u}=\left[\begin{array}{lll}x_{u} & y_{u} & 1\end{array}\right]^{T}$ and radially distorted point coordinates $\mathbf{p}_{d}=\left[\begin{array}{lll}x_{d} & y_{d} & 1\end{array}\right]^{T}$ can be written as:

$$
\left[\begin{array}{c}
x_{u} \\
y_{u} \\
1
\end{array}\right] \sim\left[\begin{array}{c}
x_{d} \\
y_{d} \\
1+\lambda r_{d}^{2}
\end{array}\right]
$$

where $\lambda$ is the distortion coefficient and $r_{d}$ is the distance of $\mathbf{p}_{d}$ to the distortion center. Here we assume that the distortion center is known and at the center of the image. We further assume that the cameras have centered principal points and square pixels. Therefore, the two unknown calibration parameters we consider here are focal length $f$ and radial distortion $\lambda$. The calibration matrix $\mathbf{K}$ can be expressed as:

$$
\mathbf{K}=\left[\begin{array}{ccc}
1 & 0 & 0 \\
0 & 1 & 0 \\
0 & 0 & w
\end{array}\right]
$$

where $w=1 / f$. Then we have the undistorted image point coordinates

$$
\mathbf{p}_{n} \sim \mathbf{K} \mathbf{p}_{u}
$$

In two-view geometry, it is well known that for uncalibrated cameras, the fundamental matrix $\mathbf{F}$ has 7 degrees of freedom, and for calibrated cameras, the essential matrix $\mathbf{E}$ has 5 degrees of freedom [12]. On the other hand, we know that each image point correspondence gives one constraint. We consider here the one-sided case where calibration matrix $\mathbf{K}^{\prime}$ and radial distortion $\lambda^{\prime}$ is fully known for the second camera. Note that the prior knowledge on the calibration of the second camera does not reduce the degrees of freedom of the epipolar geometry. In Table 1, we summarize the related minimal problems on relative pose with radial distortion for both two-sided as well as the onesided cases. We also show the number of solutions to these minimal problems in general.

\begin{tabular}{cccc}
\hline Points & Case & Two-sided & \multicolumn{1}{c}{ One-sided } \\
\hline 9 & $\mathbf{F}+\lambda+\lambda^{\prime}$ & $24[14]$ & - \\
8 & $\mathbf{F}+\lambda$ & $16[15]$ & 8 (this paper) \\
7 & $\mathbf{E}+\lambda+f$ & $68^{*}$ & 19 (this paper) \\
6 & $\mathbf{E}+\lambda$ & $52[14]$ & 26 (this paper) \\
\hline
\end{tabular}

Table 1. Minimal problems and number of solutions for relative pose with unknown radial distortion on both cameras (two-sided) and a single camera (one-sided). (*) To the best of our knowledge, this minimal problem has not been solved. The number of solutions for the $\mathbf{E}+\lambda+f$ case is based on our own initial calculation using Macaulay2 [7].

In the following sections, we describe the geometric constraints for these problems including the epipolar constraints from the point correspondences and the intrinsic constraints on the fundamental matrix and the essential matrix.

\subsection{Fundamental Matrix and Radial Distortion}

Given $n$ point correspondences, the epipolar constraints on the fundamental matrix are:

$$
\mathbf{p}_{n_{i}}^{T}(\lambda) \mathbf{F} \mathbf{p}_{n_{i}}^{\prime}=0, \quad i=1, \ldots, n .
$$


where $\mathbf{p}_{n}$ and $\mathbf{p}_{n}^{\prime}$ are corresponding undistorted image points in the first and the second image, respectively.

The singularity of the fundamental matrix $\mathbf{F}$ is enforced as:

$$
\operatorname{det}(\mathbf{F})=0 .
$$

\subsection{Essential Matrix and Radial Distortion}

Depending on whether the focal length $f$ is known for the first camera, we have two minimal cases as follows.

Radial Distortion Only. If two cameras are calibrated up to an unknown radial distortion on the first camera, given $n$ point correspondences, the epipolar constraints on the essential matrix $\mathbf{E}$ are:

$$
\mathbf{p}_{u_{i}}^{T}(\lambda) \mathbf{E}_{u_{i}}^{\prime}=0, \quad i=1, \ldots, n .
$$

Radial Distortion and Focal Length. Here we study the case where the first camera is calibrated up to an unknown focal length and an unknown radial distortion. Based on (1) and the calibration matrix $\mathbf{K}$, we can parameterize the undistorted case and normalize points as a function of $\lambda$ and $w$. Similarly, the constraints on the essential matrix are:

$$
\mathbf{p}_{u_{i}}^{T}(\lambda, w) \mathbf{E} \mathbf{p}_{u_{i}}^{\prime}=0, \quad i=1, \ldots, n .
$$

We then use the intrinsic constraints on essential matrices. The singularity of the essential matrix is enforced as:

$$
\operatorname{det}(\mathbf{E})=0 .
$$

The trace constraint, that says that the two singular values are equal, is expressed as the following constraints:

$$
2 \mathbf{E E}^{T} \mathbf{E}-\operatorname{trace}\left(\mathbf{E} \mathbf{E}^{T}\right) \mathbf{E}=0 .
$$

\section{Polynomial Solvers}

In this section, we describe the numerical schemes for solving the polynomial systems in detail. For all of these problems, the solution involves the following steps: (i) a linear elimination step [15] to reduce the number of unknowns in the polynomial system, (ii) solving the reduced polynomial system using Gröbner basis methods [6]. The methods in [6] are general, fast and stable for small-size polynomial problems. To achieve a good trade-off between speed and accuracy, we choose the basis selection strategy based on column-pivoting (instead of the more accurate but slower SVD-based scheme).

\subsection{Point Case: $\mathbf{F}+\lambda$}

We parameterize the fundamental matrix $\mathbf{F}$ as

$$
\mathbf{F}=\left[\begin{array}{lll}
f_{1} & f_{4} & f_{7} \\
f_{2} & f_{5} & f_{8} \\
f_{3} & f_{6} & f_{9}
\end{array}\right] .
$$

To fix the scale of the fundamental matrix, we assume that $f_{9} \neq 0$ and choose $f_{9}=1$.
Linear Elimination. We first look at the 8 equations in (4) given by 8 point correspondences. This gives a polynomial system in matrix form as follows:

$$
\mathbf{G x}=0,
$$

where $\mathbf{G}$ is a $8 \times 12$ coefficient matrix, and $\mathbf{x}$ is a vector of monomials $\left[\lambda f_{3}, \lambda f_{6}, f_{1}, f_{2}, f_{3}, f_{4}, f_{5}, f_{6}, f_{7}, f_{8}, \lambda, 1\right]^{T}$. Given that $\left\{f_{1}, f_{2}, f_{4}, f_{5}, f_{7}, f_{8}\right\}$ are linear in (11), we can eliminate these 6 unknowns using 6 of the 8 equations. We can further choose to eliminate one more unknown $f_{3}$ or $f_{6}$ using the remaining 2 equations. Here we choose to eliminate $f_{3}$. To achieve this, we use the two last equations to eliminate $f_{3}$ and $\lambda f_{3}$. To this end, we can rewrite the two equations as $f_{3}=g_{1}\left(f_{6}, \lambda\right)$ and $\lambda f_{3}=g_{2}\left(f_{6}, \lambda\right)$. Thus, we obtain a new polynomial system with only 2 unknowns i.e. $f_{6}$ and $\lambda$. With the singularity constraint on $\mathbf{F}$ in (5), we can then obtain a bivariate polynomial system:

$$
\begin{array}{cc}
\lambda g_{1}\left(f_{6}, \lambda\right)-g_{2}\left(f_{6}, \lambda\right) & =0, \\
\operatorname{det}\left(\mathbf{F}\left(f_{6}, \lambda\right)\right) & =0 .
\end{array}
$$

The two equations are of degree 3 and 6, respectively.

Polynomial System. By expressing the resulting polynomial system with coefficients in $\mathbb{Z}_{p}$, and using algebraic geometry tools [7], we find that there are in general 8 solutions for this problem. This size of the solution set is much smaller than two-sided cases with varying (24 solutions) and constant (16 solutions) radial distortion. To solve the polynomial system, we use the method in [6] which is based on Gröbner basis methods. First, we generate the socalled elimination template by multiplying the two equations with a set of multiplication monomials. The multiplication monomials are chosen in such a way that (i) the maximum monomial degree in the resulting polynomial equations is 7, and (ii) the highest degree of any of the two unknowns in the multiplication monomials is 4 . We then arrive at an elimination template of 12 equations and 24 monomials. Then we use a column-pivoting scheme to select the monomial basis for the $8 \times 8$ action matrix. For this problem, we find that choosing the last 9 monomials (in grevlex order) as permissible set (see [6]) gives good stability. After we construct the action matrix, solutions to the polynomial system can be extracted from the eigenvectors of the transpose of the action matrix. Once we have found solutions for $\lambda$ and $f_{6}$, the solutions of the other unknowns can be calculated linearly. Given that the polynomial system has only two unknowns, one can also solve the problem using hidden variable method.

\subsection{Point Case: $\mathbf{E}+\lambda+f$}

For this problem, instead of parameterizing the essential matrix directly, we choose to parameterize $\mathbf{F}$ and solve for $\mathbf{E}$ implicitly. The reason is that, with this implicit parameterization, we achieve a simpler elimination step. 
Linear Elimination. Similar to Section 3.1, we first use point equations in (4) given by 7 point correspondences. We use 6 of the equations to linearly eliminate $\left\{f_{1}, f_{2}, f_{4}, f_{5}, f_{7}, f_{8}\right\}$. With the remaining equation, we can rewrite $\lambda f_{6}$ as a quadratic function $h\left(f_{3}, f_{6}, \lambda\right)$ of $f_{3}, f_{6}$ and $\lambda$ such that :

$$
\lambda f_{6}-h\left(f_{3}, f_{6}, \lambda\right)=0 .
$$

Now we can write the fundamental matrix $\mathbf{F}$ as a function of $f_{3}, f_{6}$ and $\lambda$.

With the parameterization of $\mathrm{K}$ as in (2), we can express the essential matrix $\mathbf{E}$ with respect to the fundamental matrix:

$$
\mathbf{E}=\mathbf{K}(w)^{T} \mathbf{F}\left(f_{3}, f_{6}, \lambda\right) \mathbf{K}^{\prime}
$$

By substituting (15) into (8) and (9), along with (14), we obtain in total 11 equations in 4 unknowns $f_{3}, f_{6}, w$ and $\lambda$.

Polynomial System. Before verifying the number of solutions, we need to simplify the equation system further. The first observation is that there are 4 equations where $w$ is the common multiplier. This will potentially introduce a set of false solutions corresponding to $w=0$. To cope with this, we simply divide all these 4 equations by $w$ (this is done implicitly by changing the monomials in the equations). Secondly, we find that after this division step, monomials involving $w$ only appear in the 9 equations from (9) in quadratic form i.e. $w^{2}$. We therefore substitute $w^{2}$ with a new variable $z=w^{2}$. To this end, we have one quadratic equation (14), one $5^{\text {th }}$ degree equation (8) and 9 equations from (9) (3 equations of $5^{\text {th }}$ degree and 6 equations of $6^{\text {th }}$ degree).

With these simplification and using [7], we verify that in general there are 19 solutions for this system. We generate the elimination template by multiplying a set of multiplication monomials such that (i) the maximum degree of the monomials in the resulting equations is 8 , and (ii) the highest degrees of the unknowns in the multiplication monomials are $[3,4,2,4]$, respectively. The resulting elimination template is of 200 equations and 231 monomials. We observe that further reducing the size of the template by limiting the degrees in the multiplication monomials generally deteriorate the numerical stability of the solver. More trimming of the elimination template could be possible by using heuristics e.g. in [13]. To construct the action matrix, we choose the last 40 monomials as the permissible set for basis selection. Again, solutions to the polynomial system can be extracted from the eigenvectors of the transpose of the $19 \times 19$ action matrix.

\subsection{Point Case: $\mathbf{E}+\lambda$}

Similar to the fundamental matrix, we parameterize the essential matrix $\mathbf{E}$ as

$$
\mathbf{E}=\left[\begin{array}{lll}
e_{1} & e_{4} & e_{7} \\
e_{2} & e_{5} & e_{8} \\
e_{3} & e_{6} & e_{9}
\end{array}\right]
$$

and set $e_{9}=1$ to fix the scale.

Linear Elimination. From point equations in (6) given by 6 point correspondences, we have a polynomial system in matrix form as follows:

$$
\mathbf{H} \mathbf{x}=0,
$$

where $\mathbf{H}$ is a $6 \times 12$ coefficient matrix, and $\mathbf{x}$ is a vector of monomials $\left[\lambda e_{3}, \lambda e_{6}, e_{1}, e_{2}, e_{3}, e_{4}, e_{5}, e_{6}, e_{7}, e_{8}, \lambda, 1\right]^{T}$. Again, since $\left\{e_{1}, e_{2}, e_{4}, e_{5}, e_{7}, e_{8}\right\}$ are linear in (17), we can eliminate these 6 unknowns using the 6 point equations.

With the two intrinsic constraints (8) and (9), we arrive at a well-defined polynomial system of 10 equations (one from (8) and 9 from (9)) in 3 unknowns $e_{3}, e_{6}$ and $\lambda$. These equations are of degree 5 or 6 .

Polynomial System. We find that there are in general 26 solutions for this problem using [7]. In contrast, the twosided case with constant unknown radial distortion [14] has in general 52 solutions and is much more difficult to solve. To generate the elimination template, we choose the set of multiplication monomials such that (i) the maximum monomial degree in the resulting equations are up to degree 8 , and (ii) the highest degrees of the unknowns in the multiplication monomial set are $[3,4,4]$ respectively. We then obtain an elimination template of 48 equations and 70 monomials. For the basis selection step, we have chosen the permissible set to be the last 32 monomials. Solutions of $e_{3}, e_{6}$ and $\lambda$ can be extracted from the eigenvectors of the transpose of the $26 \times 26$ action matrix.

\subsection{Alternative Parameterization}

In this section, we discuss the advantage of presented parameterization over an alternative parameterization. In $[1,4]$, another way to form polynomial systems for these minimal problems is described. Let us consider the example of estimating fundamental matrix and a single radial distortion. Instead of parameterizing $\lambda$ and $\mathbf{F}$ directly, in [4], the points are lifted to 4D space with the corresponding 4 by 3 radial distortion matrix which has 11 parameters (after fixing the scale). In this way, the point equations in (4) can be written as linear equations. By parameterizing the solutions as the null space of the $n$ linear equations, we can eliminate $n$ unknowns. Thus, for the onesided 8-point case, we will have a polynomial system of 3 
unknowns. Recall that in Section 3.1, using the linear elimination scheme, we obtain a polynomial system of only 2 unknowns which facilitates the subsequent polynomial solving step. And for the other two cases with even fewer correspondences, straightforward adaption of such lifting technique yields much more involved polynomial systems than the linear elimination scheme used in this paper.

\subsection{Degenerated Cases}

It is well-known that for planar scenes, the problem of determining the fundamental matrix from two views degenerates [12]. Therefore, both one-sided 8-point and onesided 9-point for estimating fundamental matrix and radial distortion degenerate for planar scenes. On the other hand, there are two degeneracies related to our formulation. The first one is the degeneracy for planar scenes for one-sided 7 point and one-side 6 point formulation. This is caused by the rank-deficiency in the linear elimination step of our formulation. The other degeneracy relates to fixing $f_{33}$ or $e_{33}$ to be 1 , which leads to failures of the algorithms for pure translation where $f_{33}=0$ or $e_{33}=0$. Using a different parameterization e.g. null space of the linear equations could resolve these degenerates but will also lead to much more involved polynomial systems. Further works are needed to derive general and fast solvers that avoid these degeneracies.

\section{Experiments}

In this section, we first study numerical stability and noise sensitivity of the proposed solvers with synthetic data. Then, we demonstrate the usefulness of the solvers on real image data.

\subsection{Synthetic Experiments}

To simulate synthetic scenes, we randomly generate 3D points within a cube of width 1000 centered at the origin. We place the two cameras to be around 1000 units away from the origin with a random rotation pointing approximately to the origin. The length of translation between the cameras is chosen to be around 300 units. The focal lengths of the cameras are uniformly generated in the range of $[1000,2000]$. Then we generate image point correspondences by projecting the $3 \mathrm{D}$ points onto the image planes $(1000 \times 1000$ pixels $)$ of both cameras. The image point coordinates are then distorted based on (1) with radial distortion coefficients chosen randomly from the interval $[-0.2,0.2]$. Note that we specify the radial distortion with respect to normalized image point coordinates in the range $[-1,1]$. Outliers are simply image points randomly generated with a uniform distribution.
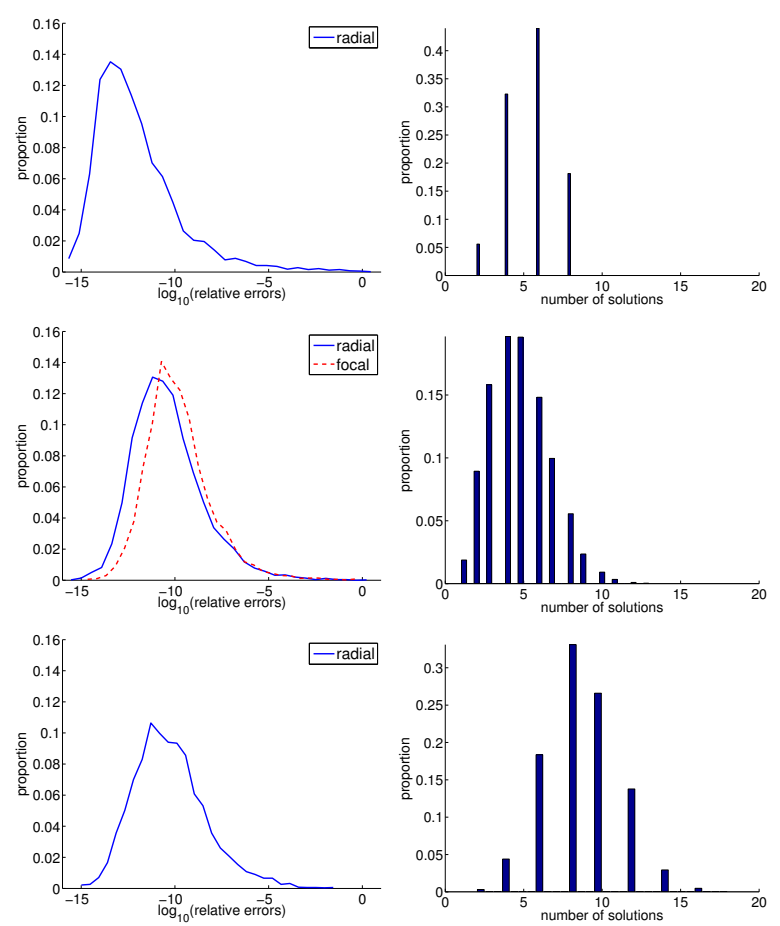

Figure 2. Left : Numerical stability of the different solvers (from top to bottom: 8 point, 7 point and 6 point minimal cases for onesided radial distortion). Error distribution compared with groundtruth radial distortion and focal length on synthetic experiments with 5000 randomly generated problems. Right: Distribution of the number of valid solutions.

Numerical Stability. We first look at the numerical stability of the solvers on noise-free data. We evaluate the solvers with relative errors between the estimated $f, \lambda$ and the ground truth. The distributions of $\log _{10}$ of the relative errors for different solvers are shown in Figure 2. We can see that both $\lambda$ and $f$ are estimated with high accuracy. For 5000 random problems, the medians of the $\log _{10}$ relative errors for $\lambda$ are -11.58, -9.54 and -10.01 for 8-point, 7-point and 6-point cases, respectively. The median of the $\log _{10}$ relative errors for $f$ estimated in the 7-point algorithm is -10.65 .

Number of Solutions. It is of interest to investigate the number of valid solutions for these minimal cases under general settings. By valid solutions, here we mean real solutions for the radial distortion $\lambda$ and positive solutions for $f$. For the 8-point problem, we see that (Figure 2, right) that there can be up to 8 real solutions in general. On the other hand, for the 7-point problem where there are in general 19 solutions, most of the time only 3-8 of the solutions are valid. As for the 6-point problem which has 26 solutions, we observe that very often, only fewer or equal to 16 of those solutions are real. The small number of valid solutions compared to the double-sided solvers further facilitates the solution verification step in RANSAC. 

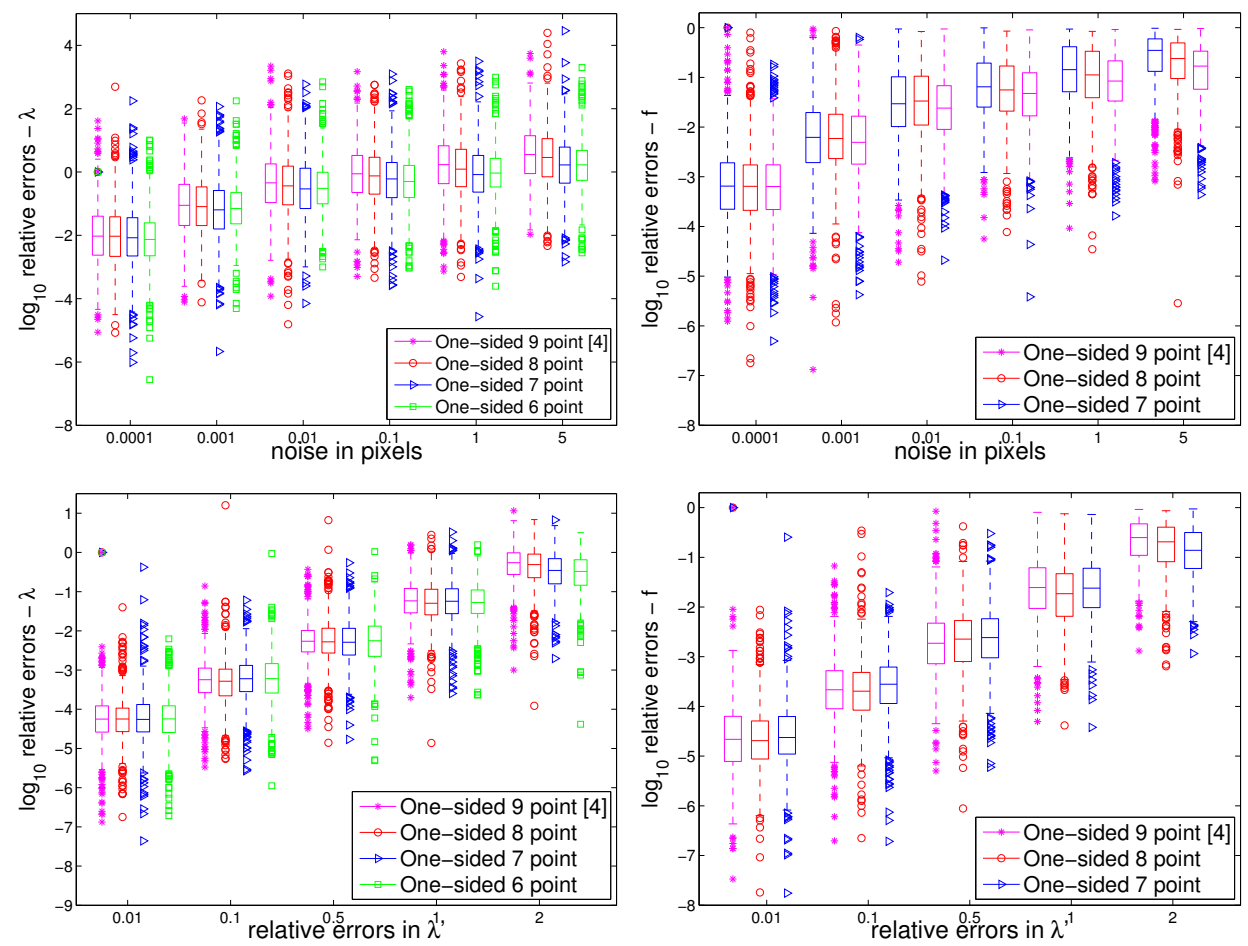

Figure 3. Top - Noise sensitivity of different solvers in estimating radial distortion (left) and focal length (right) with varying noise levels. Bottom - Sensitivity of the different solvers on calibration errors in radial distortion $\left(\lambda^{\prime}\right)$ of the second camera. Blue box specifies the 25 and 75 percentiles for the $\log _{10}$ relative errors.

Noise Sensitivity. To evaluate the solvers in the presence of noise, we perturb the image points with Gaussian noise of varying standard deviations $\sigma$. Here we are interested in the noise sensitivity of estimating the radial distortion. For small perturbations $(\sigma=0.01,0.1,0.5)$ on the image point coordinates, we see that all solvers give fairly good initial solutions to the radial distortion (Figure 3, top-left). For larger perturbations, e.g. $\sigma=1,2$, the relative errors become higher. This is similar to previous minimal solvers with radial distortion [14, 4]. These solutions from minimal configurations can be utilized in the kernel voting scheme or serve as initial solutions (after RANSAC) for bundle adjustment. While one is not aiming for better noise tolerance, the three minimal solvers proposed here perform marginally better than the non-minimal 9-point solver in $[4]^{2}$.

Estimating Focal Length. We also test and compare different solvers in estimating focal lengths with noisy measurements. For the cases where only the fundamental matrix is estimated i.e. 9-point [4] and our 8-point solver, we have extracted the focal length from the fundamental matrix using the method in [4]. We can see from Figure 3

\footnotetext{
${ }^{2}$ For all comparisons in this paper, the publicly available solvers at http://www.cvg.ethz.ch/research/distortion-in-multiple-view-geometry/ are used.
}

(top-right) that all the solvers perform similarly under settings with low noise levels. When the measurements are perturbed by noise of higher or equal to 1 pixel, our proposed solvers perform better, while requiring fewer correspondences. This further justifies the usefulness of the new minimal solvers in robust estimation settings. When comparing to the estimation of radial distortion, we observe that the estimating focal length is less sensitive to the noise i.e. the errors are lower for focal length than radial distortion under the same noise level.

Effects of Calibration Errors. In this experiment, we investigate how errors in calibrating radial distortion of the second camera affects the estimation of $\lambda$ and $f$. To study this, we add uniform random noise to the true radial distortion $\lambda^{\prime}$ of the second camera. Here, we have chosen fixed values for $\lambda$ and $\lambda^{\prime}$ such that $\lambda=-0.2$ and $\lambda^{\prime}=-0.1$. We observe (Figure 3, bottom) that for all solvers, the errors of estimating $\lambda$ increase approximately linearly with respect to the calibration errors in $\lambda^{\prime}$. On the other hand, there is no significant difference between the sensitivity of different solvers to the calibration errors.

Speed. All minimal solvers have been implemented in MATLAB. On a Macbook Air with 8GB memory and 1.8 $\mathrm{GHz}$ i5 $\mathrm{CPU}$, the average runtime for the different solvers 


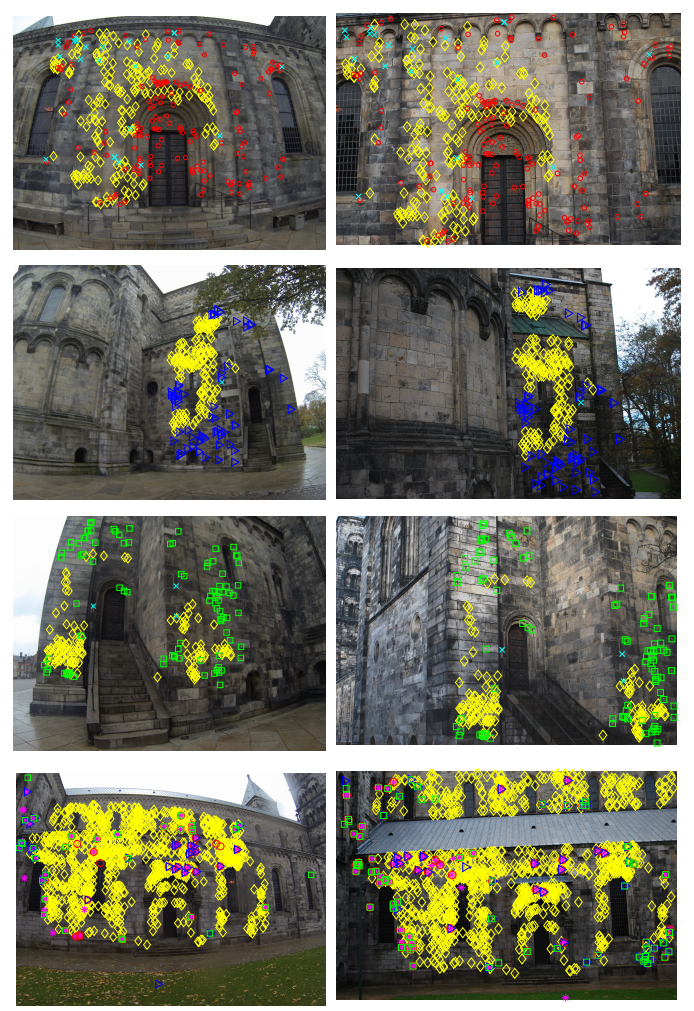

Figure 4. Example on real image pairs. Left: images taken with an GoPro-Hero3; Right: images taken with a Nikon D60 camera. Yellow diamonds are inliers obtained by the standard 7-point method. Cyan crosses are inliers only obtained by the 7-point method. The others are extra inliers obtained by the specific onesided minimal solver from the 1st to the 3rd row: one-sided 8 point, one-sided 7 point, one-sided 6 point. The last row is the comparison of inliers obtained by different one-side solvers where yellow diamonds are the common inliers.

are: $1.0 \mathrm{~ms}$ (milliseconds) for the one-sided 8-point, 8.6 $\mathrm{ms}$ for the one-sided 7-point and $3.2 \mathrm{~ms}$ for the one-sided 6-point. The solvers can be further optimized in $\mathrm{C}$ or $\mathrm{C}++$, e.g. with a more efficient construction of the coefficient matrix, which is one of the current bottleneck in MATLAB. For comparison, the one-sided 9-point method in [4] solves a cubic equation system that runs on average $0.5 \mathrm{~ms}$.

\subsection{Real Experiments}

In this section, we evaluate and validate the proposed solvers on real images. We have used an Nikon D60 camera as the fully calibrated camera in our setup. It has been verified that the Nikon D60 has very small radial distortion thus we assume that there is no radial distortion in it. We have also captured images (of resolution $3000 \times 2292$ ) with a GoPro Hero3 camera which shows significant radial distortion. To evaluate the estimation of the essential matrix as well as focal length, we calibrated the fixed focal length of

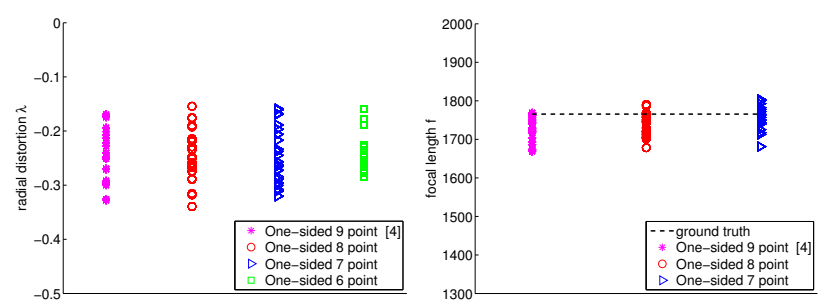

Figure 5. Estimation of radial distortion and focal length for the GoPro Hero3 camera from 25 image pairs.

the GoPro camera using the OpenCV calibration toolbox, which then serves as ground truth. For the experiments, we collected in total 25 pairs of images of different scenes with the two cameras. For each image in the dataset, we detect interest point and extract SIFT [18] features. Then for each pair of images, we obtain tentative descriptor matches based on Lowe's criterion. Given these preliminary matches, we estimate the fundamental matrix or the essential matrix using different solvers in the RANSAC loop.

In Figure 4, we show several example image pairs where we compare inliers from our minimal solvers to those obtained by the standard 7-point fundamental estimation. It can be seen that, by modeling radial distortion, we obtain more geometrically correct inliers for the image pairs. The comparison with the standard 8-point method [11] for fundamental matrix is very similar and thus is not shown here.

We then look at the gain of inliers for different minimal problems with the same number of RANSAC iterations. In Table 2, we can see that all the one-sided radial methods obtain around $60-70 \%$ more inliers than the baseline 7-point method. Our proposed minimal solvers obtain slightly more inliers $(3-5 \%)$ than the non-minimal solver in [4]. We can also see the the inlier set for different one-sided solvers are consistent with each other (Figure 4, last row).

\begin{tabular}{cccc}
\hline 9 point [4] & 8 point & 7 point & 6 point \\
\hline 1.67 & 1.71 & 1.72 & 1.70 \\
\hline
\end{tabular}

Table 2. Average ratio of inliers obtained by different one-sided radial solvers to inliers obtained by the standard 7-point algorithm.

We then study the noise sensitivity and consistency of the one-sided solvers on real images. To mitigate the effects of noise, we have also applied the kernel voting scheme of [17] to obtain better estimates for focal length and radial distortion. In Figure 5 (Left), the estimates of the radial distortion for 25 GoPro-Nikon pairs are shown. We can see that the estimates from all solvers are centered at approximately -0.25 . However, the variance of the estimates is fairly large due to the presence of noise. For the focal length (5, right), we again observe that the estimates for solvers are close to the ground truth from the calibration. In Figure 6, we show 

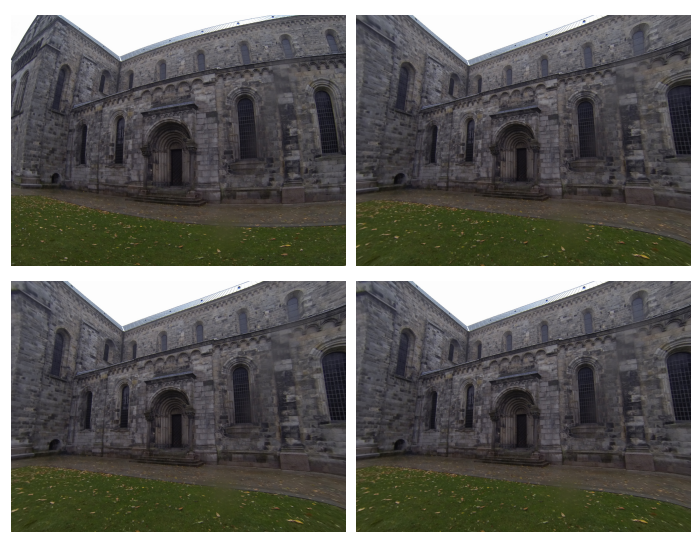

Figure 6. Example of corrected images based on the estimated $\lambda$ by different solvers. Top left: Original image. Top right, bottom left and bottom right are images corrected according to 8-point, 7-point and 6-point one-sided method respectively.

an example of corrected images according to radial distortion estimated from different one-sided methods. We can see that most of the radial distortion are correctly reduced.

\section{Conclusion}

In this paper, we study and solve three novel minimal problems for relative pose estimation with a single unknown radial distortion. We demonstrate that all these solvers are fast, numerically stable and accurate. These minimal solvers enable calibration of a camera with unknown radial distortion using another camera with known or no radial distortion. More importantly, these simpler one-sided solvers enable robust estimation of radial distortion in large-scale unordered structure from motion. In large-scale unordered structure from motion, the proposed solvers can estimate a fundamental or essential matrix robustly with respect to seed images with known or no radial distortion. Such estimation can be readily integrated with both sequential and non-sequential structure from motion methods. The study of these one-sided cases could pave the way to a deeper understanding of the previous two-sided cases as well. In particular, our solution to the one-sided essential matrix with unknown focal length and radial distortion shed light to the unsolved two-sided minimal problem of essential matrix with constant unknown focal length and radial distortion.

There are several interesting avenues for future research. Of particular theoretical interest is the critical configuration of these minimal problems. The minimal problems for essential matrix for one-sided radial with unknown distortion center is another direction to pursue.

\section{References}

[1] J. Barreto and K. Daniilidis. Fundamental matrix for cameras with radial distortion. In IEEE International Conference on
Computer Vision, Beijing, China, 2005. 1, 2, 4

[2] J. H. Brito, R. Angst, K. Köser, and M. Pollefeys. Radial distortion self-calibration. In CVPR, 2013. 2

[3] J. H. Brito, R. Angst, K. Köser, C. Zach, P. Branco, M. J. Ferreira, and M. Pollefeys. Unknown radial distortion centers in multiple view geometry problems. In Computer VisionACCV 2012, pages 136-149. Springer, 2013. 2

[4] J. H. Brito, C. Zach, K. Köser, M. J. Ferreira, and M. Pollefeys. One-sided radial fundamental matrix estimation. In BMVC, 2012. 2, 4, 6, 7

[5] M. Bujnak, Z. Kukelova, and T. Pajdla. 3d reconstruction from image collections with a single known focal length. In Computer Vision, 2009 IEEE 12th International Conference on, pages 1803-1810. IEEE, 2009. 1

[6] M. Byröd, K. Josephson, and K. Åström. Fast and stable polynomial equation solving and its application to computer vision. Int. Journal of Computer Vision, 84(3):237-255, 2009. 3

[7] D. Eisenbud, D. R. Grayson, E. S. Michael, and B. Sturmfels, editors. Computations in algebraic geometry with Macaulay 2. Springer-Verlag, 2001. 2, 3, 4

[8] M. A. Fischler and R. C. Bolles. Random sample consensus: a paradigm for model fitting with applications to image analysis and automated cartography. Communications of the ACM, 24(6):381-95, 1981. 2

[9] A. W. Fitzgibbon. Simultaneous linear estimation of multiple view geometry and lens distortion. In Proc. of Computer Vision and Pattern Recognition Conference, pages 125-132, 2001. 1, 2

[10] R. Hartley and S. B. Kang. Parameter-free radial distortion correction with center of distortion estimation. Pattern Analysis and Machine Intelligence, IEEE Transactions on, 29(8):1309-1321, 2007. 1

[11] R. I. Hartley. In defense of the eight-point algorithm. Pattern Analysis and Machine Intelligence, IEEE Transactions on, 19(6):580-593, 1997. 7

[12] R. I. Hartley and A. Zisserman. Multiple view geometry in computer vision. Cambridge university press, 2003. 1, 2, 5

[13] Z. Kukelova, M. Bujnak, and T. Pajdla. Automatic generator of minimal problem solvers. In Proc. 10th European Conf. on Computer Vision, Marseille, France, 2008. 4

[14] Z. Kukelova, M. Byröd, K. Josephson, T. Pajdla, and K. Åström. Fast and robust numerical solutions to minimal problems for cameras with radial distortion. Computer Vision and Image Understanding, 114(2):234-244, 2010. 1, 2, 4, 6

[15] Z. Kukelova and T. Pajdla. A minimal solution to the autocalibration of radial distortion. In In Proc. Conf. Computer Vision and Pattern Recognition, 2007. 1, 2, 3

[16] Z. Kukelova and T. Pajdla. Two minimal problems for cameras with radial distortion. In OMNIVIS, 2007. 1

[17] H. Li and R. Hartley. A non-iterative method for correcting lens distortion from nine point correspondences. OMNIVIS 2005, 2005. 2, 7

[18] D. G. Lowe. Object recognition from local scale-invariant features. In Proc. 7th Int. Conf. on Computer Vision, Kerkyra, Greece, pages 1150-1157, 1999. 7 